\title{
The Prognostic Impact of Dose-attenuated R-CHOP Therapy for Elderly Patients with Diffuse Large B-cell Lymphoma
}

\author{
Akira Tanimura ${ }^{1}$, Risen Hirai ${ }^{1}$, Miki Nakamura ${ }^{2}$, Masataka Takeshita ${ }^{1}$, \\ Shotaro Hagiwara ${ }^{2,3}$ and Akiyoshi Miwa ${ }^{1,2}$
}

\begin{abstract}
:
Objective Although R-CHOP (rituximab, cyclophosphamide, vincristine, doxorubicin, and prednisone) is a standard therapy for diffuse large B-cell lymphoma (DLBCL), the optimal dose for elderly patients remains unclear.

Methods and Patients We retrospectively verified our R-CHOP dose-attenuation system implemented from 2005 for DLBCL patients. Among the 115 DLBCL patients treated during 2001-2010, 33 patients treated during 2001-2005 received R-CHOP doses adjusted according to physicians' decisions (PHY group). Eightytwo patients treated after 2005 received adjusted R-CHOP doses according to a unified dose-attenuation system (UNI group). Patients aged $<60,60-69,70-79$, and $\geq 80$ years received the standard R-CHOP, $100 \%$ R$\mathrm{CHO}+\mathrm{P}\left(50 \mathrm{mg} / \mathrm{m}^{2}\right), 100 \% \mathrm{R}+75 \% \mathrm{CHO}+\mathrm{P}\left(40 \mathrm{mg} / \mathrm{m}^{2}\right)$, and $100 \% \mathrm{R}+50 \% \mathrm{CHO}+\mathrm{P}\left(30 \mathrm{mg} / \mathrm{m}^{2}\right)$, respectively. We compared the responses, survival, and treatment cessation between the PHY and UNI groups.

Results The patients' characteristics between both groups were closely comparable. All PHY patients received randomly adjusted R-CHOP doses; $94 \%$ of UNI patients received scheduled doses. The complete response rates differed significantly between the UNI (77\%) and PHY patients $(50 \%)(\mathrm{p}=0.011)$. The two-year event-free survival rates were 50\% and $32 \%$ in the UNI and PHY groups, respectively ( $\mathrm{p}=0.0083$ ). The twoyear OS rates were $77 \%$ and $72 \%$ in the UNI and PHY group ( $\mathrm{p}=0.16)$. Among the patients aged $>70$ years $(\mathrm{n}=59)$ overall survival was shorter in the PHY group $(62 \%)$ than in the UNI group $(72 \% ; \mathrm{p}=0.02)$. The UNI group received higher anti-tumor agent doses than the PHY group. The therapy discontinuation rates were $5 \%$ in the UNI group and $24 \%$ in the PHY group.

Conclusion Carrying out unified dose reduction may improve the efficacy and prognosis among elderly DLBCL patients.
\end{abstract}

Key words: DLBCL, R-CHOP, elderly, comorbidities, dose-attenuation

(Intern Med 57: 3521-3528, 2018)

(DOI: 10.2169/internalmedicine.0990-18)

\section{Introduction}

Diffuse large B-cell lymphoma (DLBCL) is the most frequent subtype of non-Hodgkin lymphoma (NHL) (1-3). Population aging in developed nations has led to an increased incidence of DLBCL, and currently the median age of the patients at diagnosis is $>65$ years $(1,3)$. Despite of this tendency of DLBCL occurrence in elderly people, the therapeutic approaches for DLBCL in this population remain challenging (4-6). Elderly people often have comorbidities and impaired organ functions due to several diseases and various physiological changes. Accordingly, issues of chemokinetics and chemodynamics in the elderly differ from those in younger patients, and they may lead to the more frequent development of adverse effects or events related to

${ }^{1}$ Department of Hematology, Tokyo-Kita Medical Center, Japan, ${ }^{2}$ Division of Hematology, National Center for Global Health and Medicine, Japan and ${ }^{3}$ Division of Hematology, Tokyo Women's Medical University, Japan

Received: February 9, 2018; Accepted: May 13, 2018; Advance Publication by J-STAGE: August 10, 2018

Correspondence to Dr. Akira Tanimura, akira.tanimura@h3.dion.ne.jp 
anti-tumor drug therapy among older patients (7). Notwithstanding the fact that the current standard therapies were established through clinical trials that yielded promising results in younger patients without any severe comorbidities, it remains unknown whether the same drug doses and treatment intervals can be safely applied to the elderly population. Accordingly, physicians often tend to administer doseattenuated chemotherapy to elderly patients, who generally present with comorbidities affecting their performance status (8).

The introduction of the immunotherapeutic drug rituximab to conventional chemotherapeutic agents led to the development of R-CHOP (rituximab (R), cyclophosphamide (CY), vincristine (VCR), doxorubicin (DOX), prednisone (PSL)). This combination therapy comprising $\mathrm{R}$ and conventional CHOP yielded better cure rates of DLBCL in both younger and elderly patients, exemplified by the finding that the complete response (CR) and overall survival (OS) rates of elderly patients receiving a curative dose of R-CHOP were not inferior to those of younger patients $(9,10)$. However, potentially curative, anthracycline-containing therapies might be too toxic for very elderly patients ( $\geq 80$ years), unfit patients, and those with severe comorbidities, where the risks surpass the benefits of chemotherapy $(11,12)$. Although some trials have reported the safe administration of attenuated chemotherapeutic regimens to very elderly and/or unfit patients, these studies reported inferior survival outcomes relative to those observed in younger or fit patients $(13,14)$. Furthermore, the methods of attenuation and the outcomes

Table 1. Dose Attenuation by Patients' Age.

\begin{tabular}{lccccc}
\hline \multicolumn{1}{c}{ Age } & CY & DOX & VCR & PSL & R \\
\hline$\leq 59$ & $100 \%$ & $100 \%$ & $100 \%$ & $100 \mathrm{mg} /$ body & $100 \%$ \\
$60-69$ & $100 \%$ & $100 \%$ & $100 \%$ & $50 \mathrm{mg} / \mathrm{m}^{2}$ & $100 \%$ \\
$70-79$ & $75 \%$ & $75 \%$ & $75 \%$ & $40 \mathrm{mg} / \mathrm{m}^{2}$ & $100 \%$ \\
$\geq 80$ & $50 \%$ & $50 \%$ & $50 \%$ & $30 \mathrm{mg} / \mathrm{m}^{2}$ & $100 \%$ \\
\hline
\end{tabular}

CY: cyclophosphamide, DOX: doxorubicin, PSL: prednisone, R: rituximab, VCR: vincristine of the reduced therapies have not yet been sufficiently verified in elderly DLBCL patients. In this retrospective study, we aimed to verify the efficacy of our unified doseattenuation system for R-CHOP. In this system, which was implemented in 2005, age, performance status (PS), comorbidities and laboratory findings were taken into consideration. Through comparisons of the outcomes of patients treated before and after implementation, we could confirm the adaptability of this system.

\section{Materials and Methods}

We analyzed 115 consecutive patients with DLBCL who were treated at our institute from September 2001 (when rituximab was first approved in Japan) to December 2010. From September 2001 to August 2005, 33 patients received dose-adjusted R-CHOP according to the physician's discretion (PHY group). From September 2005, 82 patients received R-CHOP according to the unified dose-attenuation system (UNI group).

In the UNI group, patients aged $<60$ years received the standard R-CHOP dose [R, $375 \mathrm{mg} / \mathrm{m}^{2} ; \mathrm{CY}, 750 \mathrm{mg} / \mathrm{m}^{2}$; DOX, $50 \mathrm{mg} / \mathrm{m}^{2}$; VCR, $1.4 \mathrm{mg} / \mathrm{m}^{2}$ (maximum: $2.0 \mathrm{mg} /$ body); PSL, $100 \mathrm{mg} /$ body]. In patients aged $\geq 60$ years, the doses of CY, DOX, VCR, PSL, and R were attenuated as shown in Table 1; the doses of CY, DOX, and VCR were also adjusted according to the laboratory findings (Table 2). These attenuation criteria were determined at our institute after a full review and discussion of standard pharmacology texts, pharmaceutical drug information, and data of published related studies and reviews retrieved from PubMed. The dose of each agent was adjusted during the first cycle of R-CHOP. After the second cycle, the doses could be adjusted at the physician's discretion to address improvements in comorbidities, PS, and damage from tumor burdens, or toxicities from the previous treatment. If the severity of organ damage, grade of PS, and general condition were judged as inadequate to initiate CY, DOX, or VCR, then a pre-phase therapeutic regimen comprising high-dose dex-

Table 2. Dose Attenuation by Organ Dysfunctions.

\begin{tabular}{|c|c|c|c|c|c|c|}
\hline $\begin{array}{l}\text { Cytotoxic } \\
\text { agents }\end{array}$ & $\begin{array}{l}\text { Cause of } \\
\text { reduction }\end{array}$ & $100 \%$ & $75 \%$ & $50 \%$ & $25 \%$ & $0 \%$ \\
\hline \multirow[t]{2}{*}{$\mathrm{CY}$} & $\begin{array}{c}\text { Liver } \\
\text { damage }\end{array}$ & $\begin{array}{l}\text { T.Bil<3.0 mg/dL and } \\
\text { AST } \leq 179 \mathrm{IU} / \mathrm{L}\end{array}$ & $\begin{array}{c}\text { T.Bil } 3.1-4.9 \mathrm{mg} / \mathrm{dL} \\
\text { or AST>179 IU/L }\end{array}$ & - & - & T.Bil $\geq 5 \mathrm{mg} / \mathrm{dL}$ \\
\hline & CCR & $\mathrm{CCR} \geq 10 \mathrm{~mL} / \mathrm{min}$ & - & $\mathrm{CCR}<10 \mathrm{~mL} / \mathrm{min}$ & - & - \\
\hline \multirow[t]{2}{*}{ DOX } & $\begin{array}{c}\text { Liver } \\
\text { damage }\end{array}$ & $\begin{array}{c}\text { T.Bil }<1.2 \mathrm{mg} / \mathrm{dL} \text { and } \\
\text { AST and ALT }<2 \text { XULNR }\end{array}$ & $\begin{array}{c}\text { AST or ALT 2-3X of } \\
\text { ULNR }\end{array}$ & $\begin{array}{c}\text { T.Bil } 1.2-2.9 \mathrm{mg} / \mathrm{dL} \text { or } \\
\text { AST of } \\
\text { ALT }>3 \text { XULNR }\end{array}$ & $\begin{array}{l}\text { T.Bil 3.0-4.9 } \\
\text { mg/dL }\end{array}$ & $\mathrm{T} . \mathrm{Bil} \geq 5 \mathrm{mg} / \mathrm{dL}$ \\
\hline & CCR & $\mathrm{CCR} \geq 10 \mathrm{~mL} / \mathrm{min}$ & $\mathrm{CCR}<10 \mathrm{~mL} / \mathrm{min}$ & - & - & \\
\hline \multirow[t]{2}{*}{ VCR } & $\begin{array}{l}\text { Liver } \\
\text { damage }\end{array}$ & $\begin{array}{l}\text { T.Bil }<1.5 \mathrm{mg} / \mathrm{dL} \text { and } \\
\text { AST }<60 \mathrm{IU} / \mathrm{L}\end{array}$ & - & $\begin{array}{l}\text { T.Bil } 1.5-3.0 \mathrm{mg} / \mathrm{dL} \text { or } \\
\text { AST } 60-179 \mathrm{IU} / \mathrm{L}\end{array}$ & $\begin{array}{c}\text { T.Bil 3.1-4.9 } \\
\text { mg/dL }\end{array}$ & $\begin{array}{c}\text { T.Bil } \geq 5 \mathrm{mg} / \mathrm{dL} \text { or } \\
\text { AST } \geq 180 \mathrm{IU} / \mathrm{L}\end{array}$ \\
\hline & CCR & any & - & - & - & - \\
\hline
\end{tabular}

ALT: alanine aminotransferase, AST: aspartate aminotransferase, CCR: creatinine clearance, CY: cyclophosphamide, LDH: lactate dehydrogenase, DOX: doxorubicin, PHY: physicians' decision group, PS: performance status, R-IPI: Revised International Prognostic Index, T.Bil: total bilirubin, ULNR: upper limit of normal range, UNI: unified dose-attenuation group, VCR: vincristine 
Table 3. Patients' Characteristics.

\begin{tabular}{|c|c|c|c|c|}
\hline & All (115) & PHY (33) & UNI (82) & $\mathrm{p}$ value \\
\hline Median age (median, range) & $70(38-91)$ & $67(47-91)$ & $71.5(38-88)$ & 0.12 \\
\hline$<70$ y.o. $/ \geq 70$ y.o. & $56 / 59$ & $20 / 13$ & $36 / 46$ & 0.11 \\
\hline $\operatorname{Sex}(M / F)$ & $56 / 59$ & $21 / 12$ & $35 / 47$ & 0.04 \\
\hline Stage (I,II/ III,IV) & $34 / 81$ & $8 / 25$ & $26 / 56$ & 0.43 \\
\hline PS $0-1 / 2-4 / \mathrm{NA}$ & $73 / 31 / 11$ & $17 / 10 / 6$ & $56 / 21 / 5$ & 0.33 \\
\hline Extranodal lesions $0-1 />2 / \mathrm{NA}$ & $71 / 43 / 1$ & $26 / 6 / 1$ & $45 / 37 / 0$ & 0.01 \\
\hline LDH (IU/L, median, range) & $289(134-3,033)$ & $287(134-1,267)$ & $291(144-3,033)$ & 0.94 \\
\hline R-IPI (very good/good/poor/NA) & $5 / 41 / 68 / 1$ & $2 / 10 / 20 / 1$ & $3 / 31 / 48 / 0$ & 0.71 \\
\hline Albumin (g/dL, median, range) & $3.4(1.5-4.8)$ & $3.5(1.6-4.8)$ & $3.3(1.5-4.7)$ & 0.048 \\
\hline Creatinine (mg/dL, median, range) & $0.73(0.31-13.22)$ & $0.73(0.4-3.27)$ & $0.72(0.31-13.22)$ & 0.29 \\
\hline T.Bil (mg/dL, median, range) & $0.7(0.2-9.5)$ & $0.6(0.3-6.2)$ & $0.7(0.2-9.5)$ & 0.034 \\
\hline AST (IU/L, median, range) & $25(9-728)$ & $31(9-665)$ & $24(12-728)$ & 0.27 \\
\hline ALT (IU/L, median, range) & $17(5-795)$ & $15(5-795)$ & $17(6-554)$ & 0.64 \\
\hline CCI (median, range) & $2(0-7)$ & $2(0-9)$ & $3(0-9)$ & 0.74 \\
\hline CCI $0-2 / \geq 3$ & $60 / 55$ & $15 / 18$ & $45 / 37$ & 0.41 \\
\hline
\end{tabular}

ALT: alanine aminotransferase, AST: aspartate aminotransferase, CCI: Charlson Comorbidity Index, R-IPI: Revised International Prognostic Index, LDH: lactate dehydrogenase, NA: not available, PHY: physicians' decision group, PS: performance status, T.Bil: total bilirubin, UNI: unified dose-attenuation group

amethasone (20-40 mg daily, 4 consecutive days) was administered, and a systemically attenuated first cycle of RCHOP was initiated after improvements in any organ damage, PS and the patients' general condition.

Acyclovir and trimethoprim-sulfamethoxazole were prescribed as a prophylaxis against infection for elderly patients ( $\geq 60$ year-old). Levofloxacin and fluconazole were prescribed for the patients with low PS and neutropenia. Patients aged $\geq 60$ years also received granulocyte-colony stimulating factor (G-CSF) during neutropenic period. Patients with diabetes mellitus were hospitalized and received subcutaneous or intravenous injections of insulin while receiving steroid therapy.

All patients provided their written informed consent to receive each regimen, and treatment was administered according to the principles of the Declaration of Helsinki and this study was approved by the institutional ethics committee.

According to a statistical analysis, differences between the two groups were examined using Fisher's exact test for categorical variables and the Mann-Whitney U-test for continuous variables. The overall survival (OS) duration was calculated from the initiation of therapy to the last confirmation that the patient remained alive or had died from any cause. The event-free survival (EFS) interval was calculated from the initiation of treatment to the onset of a clinically significant event: death from any cause, relapse, progression, or chemotherapy discontinuation. Survival curves were prepared using the Kaplan-Meier method and the significance of difference of survival curves were analyzed using the logrank test. A logistic regression model was used for the multivariate analysis of the prognostic factors for CR rate. A Cox proportional hazard regression model were used for the multivariable analysis of the prognostic factors for survival. All statistical analyses were performed using the EZR software program (Saitama Medical Center, Jichi Medical Uni- versity, Saitama, Japan), a graphical user interface of R (version 3.0.2; The R Foundation for Statistical Computing, Vienna, Austria) (15).

\section{Results}

\section{Patients' characteristics}

The patients in this study had a median age of 70 (range, 38-91) years. In this cohort, $70.4 \%$ of patients had stage III or IV DLBCL, $40.4 \%$ had an International Prognostic Index (IPI) score of $0-2$, and $70.2 \%$ had an Eastern Cooperative Oncology Group PS of 0 or 1 . Additionally, $50.5 \%$ of patients had a low (i.e., below normal range) serum albumin level, and $48 \%$ had a high Charlson Comorbidity Index (CCI) score of $>2$. Patients in the PHY and UNI groups had similar characteristics without the number of extranodal lesions (Table 3). PET-CT could not been routinely used for the evaluation of clinical stage in the PHY group, but PETCT evaluation was performed in a large part of patients of UNI group. That was the reason why extranodal lesions were detected more frequently in the UNI group than in the PHY group (Table 3).

\section{Response rates}

The complete response (CR) rates were $77 \%$ and $50 \%$ in the UNI and PHY groups, respectively ( $\mathrm{p}=0.011)$. A univariate analysis identified a low IPI score, low lactate dehydrogenase level, better PS, and UNI group as significant indicators of a better CR prognosis, whereas only low IPI score retained significance in a multivariate analysis (Table 4). Among the patients aged $\geq 70$ years $(n=59)$, those in the UNI and PHY groups had CR rates of $81 \%$ and $13 \%$, respectively $(\mathrm{p}=0.0004)$. In the PHY group, patients aged $\geq 70$ years had a lower CR rate $(66 \%$ vs. $13 \%, \mathrm{p}=0.03)$. By con- 
Table 4. Univariate and Multivariate Analyses of CR Rate.

\begin{tabular}{|c|c|c|c|c|c|c|}
\hline \multirow{2}{*}{ Factors } & \multirow{2}{*}{ Group } & \multirow{2}{*}{$\mathrm{CR}$ rate } & \multicolumn{2}{|c|}{ univariate } & \multicolumn{2}{|c|}{ multivariate } \\
\hline & & & OR $(95 \% \mathrm{CI})$ & $\mathrm{p}$ value & OR $(95 \% \mathrm{CI})$ & $\mathrm{p}$ value \\
\hline \multirow[t]{2}{*}{ Age } & $<70$ & $70 \%$ & 1 & 0.93 & ND & - \\
\hline & $\geq 70$ & $71 \%$ & $0.96(0.42-2.23)$ & 0.93 & ND & - \\
\hline \multirow[t]{2}{*}{ Albumin } & $<3.5 \mathrm{~g} / \mathrm{dL}$ & $65 \%$ & 1 & 0.26 & ND & - \\
\hline & $\geq 3.5 \mathrm{~g} / \mathrm{dL}$ & $75 \%$ & $0.61(0.26-1.44)$ & 0.26 & ND & - \\
\hline \multirow[t]{2}{*}{ Treatment group } & PHY & $50 \%$ & 1 & 0.011 & 1 & 0.062 \\
\hline & UNI & $77 \%$ & $0.3(0.12-0.76)$ & 0.011 & $0.24(0.09-0.67)$ & 0.062 \\
\hline \multirow[t]{2}{*}{$\mathrm{CCI}$} & $0-2$ & $78 \%$ & 1 & 0.12 & 1 & 0.278 \\
\hline & $\geq 3$ & $62 \%$ & $1.97(0.83-4.7)$ & 0.12 & $1.69(0.66-4.35)$ & 0.278 \\
\hline \multirow[t]{2}{*}{ LDH } & high $>$ ULNR & $61 \%$ & 1 & 0.022 & ND & - \\
\hline & normal & $82 \%$ & $0.34(0.13-0.85)$ & 0.022 & ND & - \\
\hline \multirow[t]{2}{*}{ IPI } & $0-2$ & $84 \%$ & 1 & 0.001 & 1 & 0.0012 \\
\hline & $3-5$ & $60 \%$ & $3.52(1.35-9.19)$ & 0.001 & $3.76(1.33-10.6)$ & 0.0012 \\
\hline \multirow[t]{2}{*}{ PS } & $0-1$ & $80 \%$ & 1 & 0.0022 & ND & - \\
\hline & $2-4$ & $46 \%$ & $4.73(1.75-12.8)$ & 0.0022 & ND & - \\
\hline
\end{tabular}

CCI: Charlson Comorbidity Index, CR: complete remission, IPI: International Prognostic Index, LDH: lactate dehydrogenase, OR: odds ratio, PHY: physicians' decision group, PS: performance status, ULNR: upper limit of normal range, UNI: unified dose-attenuation group
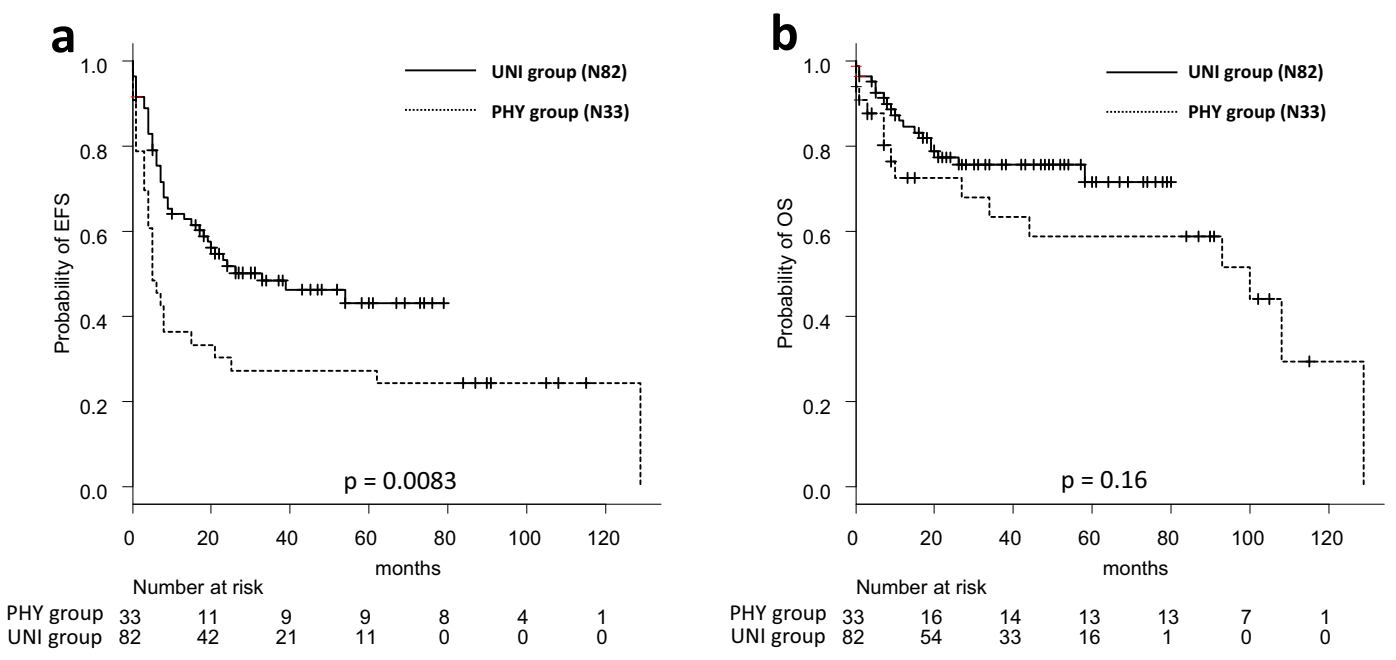

Figure 1. Event-free and overall survival according to the treatment group Event-free survival. (a) and overall survival (b) in the 115 patients. 2-year EFS are 50\% (UNI) and 32\% (PHY), and 2-year OS are $77 \%$ (UNI) and $72 \%$ (PHY). PHY: physicians' decision group, UNI: unified dose-attenuation group

trast, the patients' age did not have any effect on the CR rate $(<70$ years, $71 \%$ vs. $70-79$ years, $83 \%$ vs. $\geq 80$ years, $79 \% ; \mathrm{p}=0.56)$.

\section{Survival}

During a median follow-up of 26 months, the 2-year EFS rates were $50 \%$ and $32 \%$ in the UNI and PHY groups, respectively ( $\mathrm{p}=0.0083$, Fig. 1a). The 2-year OS rates were $77 \%$ and $72 \%$ in the UNI and PHY group ( $\mathrm{p}=0.16$, Fig. $1 \mathrm{~b}$ ). A multivariate analysis identified the UNI group [hazard ratio $(\mathrm{HR})=0.50, \mathrm{p}=0.0072$ ] as a positive prognostic factor for EFS, and a high CCI score $(\geq 3)$ and a high IPI score $(\geq 2)$ as negative prognostic factors $(\mathrm{HR}=1.77, \mathrm{p}=0.0039$ and $\mathrm{HR}=$ $2.75, \mathrm{p}=0.0005$, respectively). Additionally, a normal albu- min value $(\geq 3.5 \mathrm{~g} / \mathrm{dL})$ was identified as a better prognostic factor for OS (HR=0.44, $\mathrm{p}=0.047)$, and a high IPI score $(\geq 2)$ was identified as a poor prognostic factor for OS $(\mathrm{HR}=3.72$, $\mathrm{p}=0.010$ ) (Table 5).

Among the patients aged $\geq 70$ years $(n=59)$, the UNI group had superior rates in both EFS and OS compared with the PHY group $(50 \%$ vs. $0 \%$ for 2-year EFS, p=0.0006; $72 \%$ vs. $62 \%$ for 2 -year OS, p=0.02; Fig. 2). In the PHY group, patients aged $\geq 70$ years had a lower CR rate $(66 \%$ vs. $13 \%, \mathrm{p}=0.03$ ) and tended to have a shorter OS relative to the younger patients, although the latter difference was not statistically significant ( $78 \%$ vs. $62 \%$, p=0.084; Fig. 3a). By contrast, the patients' age did not have any effect on 2year OS in the UNI group ( $<70$ years, $83 \%$ vs. $\geq 70$ years, 
Table 5. Univariate and Multivariate Analyses of EFS and OS.

\begin{tabular}{|c|c|c|c|c|c|c|c|c|}
\hline & \multicolumn{4}{|c|}{ EFS } & \multicolumn{4}{|c|}{ OS } \\
\hline & \multicolumn{2}{|c|}{ univariate } & \multicolumn{2}{|c|}{ multivariate } & \multicolumn{2}{|c|}{ univariate } & \multicolumn{2}{|c|}{ multivariate } \\
\hline & HR $(95 \%$ CI $)$ & $\mathrm{p}$ value & HR $(95 \%$ CI $)$ & $\mathrm{p}$ value & HR $(95 \%$ CI $)$ & $\mathrm{p}$ value & HR $(95 \%$ CI $)$ & $\mathrm{p}$ value \\
\hline Age $(\geq 70)$ & $1.48(0.91-2.4)$ & 0.12 & ND & - & $1.74(0.85-3.55)$ & 0.131 & ND & - \\
\hline $\begin{array}{l}\text { Albumin } \\
(\geq 3.5 \mathrm{~g} / \mathrm{dL})\end{array}$ & $0.68(0.42-1.11)$ & 0.121 & ND & - & $0.2746(0.13-0.59)$ & 0.001 & $0.44(0.19-0.99)$ & 0.047 \\
\hline $\begin{array}{l}\text { Treatment group } \\
\text { (UNI) }\end{array}$ & $0.52(0.37-0.86)$ & 0.011 & $0.50(0.30-0.83)$ & 0.0072 & $0.59(0.28-1.25)$ & 0.167 & $0.50(0.24-10.8)$ & 0.079 \\
\hline $\mathrm{CCI}(\geq 3)$ & $2.29(1.36-3.89)$ & 0.0019 & $1.77(1.03-3.03)$ & 0.0039 & $3.77(1.62-8.75)$ & 0.002 & $2.22(0.92-5.35)$ & 0.076 \\
\hline LDH (normal) & $0.5(0.3-0.83)$ & 0.0079 & ND & - & $0.32(0.14-0.7)$ & 0.0048 & ND & - \\
\hline IPI $(\geq 3)$ & $2.93(1.68-5.1)$ & 0.00015 & $2.75(1.55-4.86)$ & 0.0005 & $5.54(2.13-14.43)$ & 0.00045 & $3.72(1.38-10.0)$ & 0.010 \\
\hline PS $(\geq 2)$ & $3.01(1.78-5.1)$ & 0.00004 & ND & - & $3.43(1.62-7.28)$ & 0.0013 & ND & - \\
\hline
\end{tabular}

CCI: Charlson Comorbidity Index, CI: confidence interval, EFS: event-free survival, HR: hazard ratio, IPI: International Prognostic Index, LDH: lactate dehydrogenase, ND: not done, OS: overall survival, PS: performance status, UNI: unified dose-attenuation group
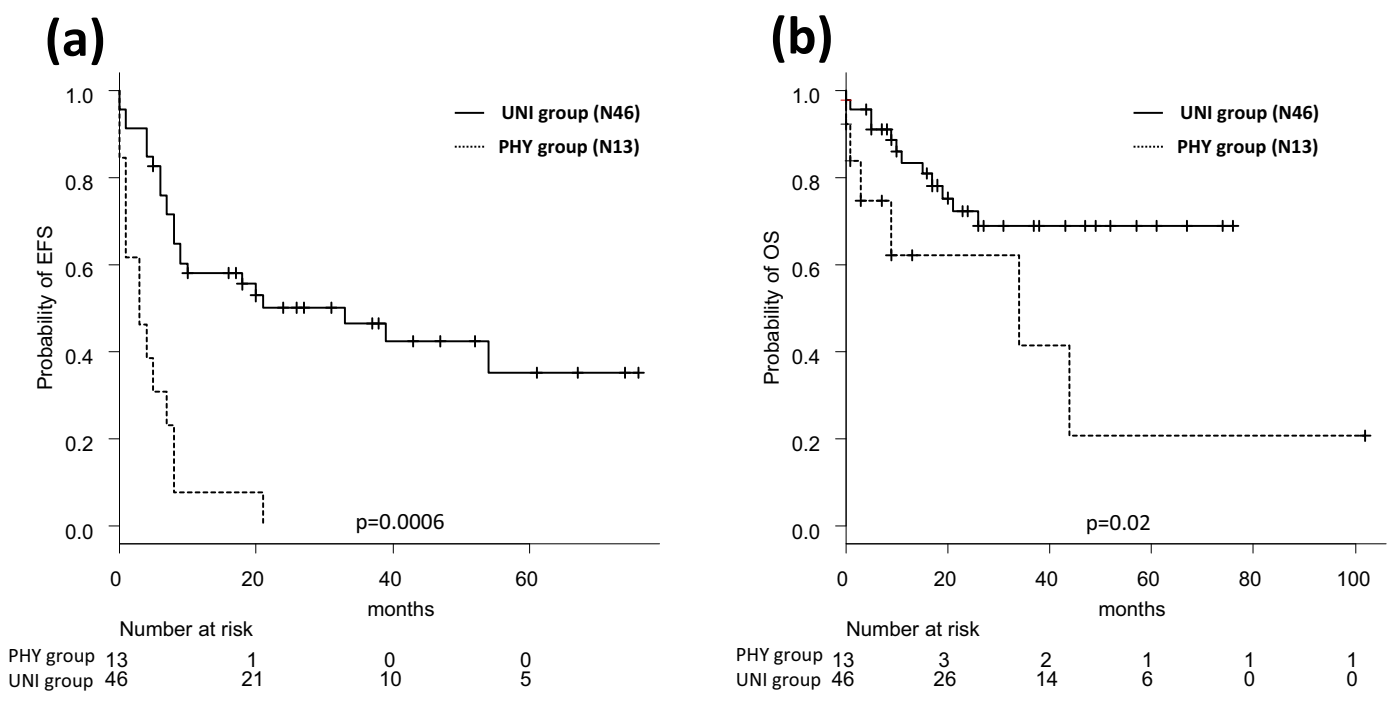

Figure 2. Event-free and overall survivals of 59 patients aged $\geq 70$ years according to the treatment group. Event-free survival (a) and overall survival (b) in the 59 patients older than 70 years. 2-year EFS are 50\% (UNI) and 0\% (PHY), and 2-year OS are 72\% (UNI) and 62\% (PHY). PHY: physicians' decision group, UNI: unified dose-attenuation group

$72 \%, \mathrm{p}=0.31$; Fig. 3b).

\section{Clinical course and dosages of cytotoxic agents}

In the UNI group, the attenuation criteria for cytotoxic agents and prednisone were met in $94 \%$ and $79 \%$ of cases, respectively. The actual doses of CY, DOX, and VCR did not differ from the standard R-CHOP doses given to patients aged $<70$ years, but they differed significantly among the patients aged $\geq 70$-years (Supplementary material 1 ). In the PHY group, 6 patients did not receive any doses of cytotoxic agents. One patient was in his eighty, and other 5 patients in their seventies. Two patients were in PS3, besides three patients were in PS4. Their IPI scores were full. One patient with PS 1 in a limited stage of lymphoma in his tonsils refused to receive cytotoxic agents. In the UNI group, 16 patients with an extremely poor general condition (i.e., severe renal or hepatic damage and/or a poor PS) received pre-phase dexamethasone therapy, and 15 could proceed to
R-CHOP therapy after achieving improvement of general condition. Among these 15 patients, seven patients attained $\mathrm{CR}$, there attained PR, four discontinued R-CHOP, and one died during R-CHOP therapy.

Disease progression during treatment, therapy discontinuation, and death during treatment were observed in $15.2 \%$ and $9.8 \%, 24.2 \%$ and $4.9 \%$, and $3.0 \%$ and $4.9 \%$ of the patients in the PHY and UNI groups, respectively (Supplementary material 2).

Nineteen patients $(23 \%)$ in the UNI group died during a median follow-up of 15 months, and 15 (45\%) in the PHY group died during a median follow-up of 29 months. Twelve deaths $(15 \%)$ in the UNI group and $8(24 \%)$ in the PHY group were attributed to lymphoma. Five second primary malignancies were observed (one case each of colon cancer and breast cancer in the PHY group; one case of lung cancer and two of myelodysplastic syndrome in the UNI group), and they were considered to be responsible for four 
(a)

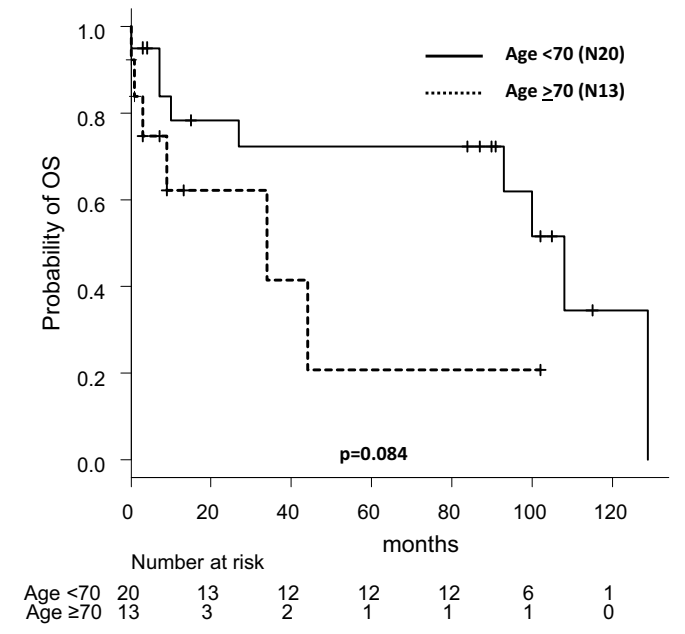

(b)

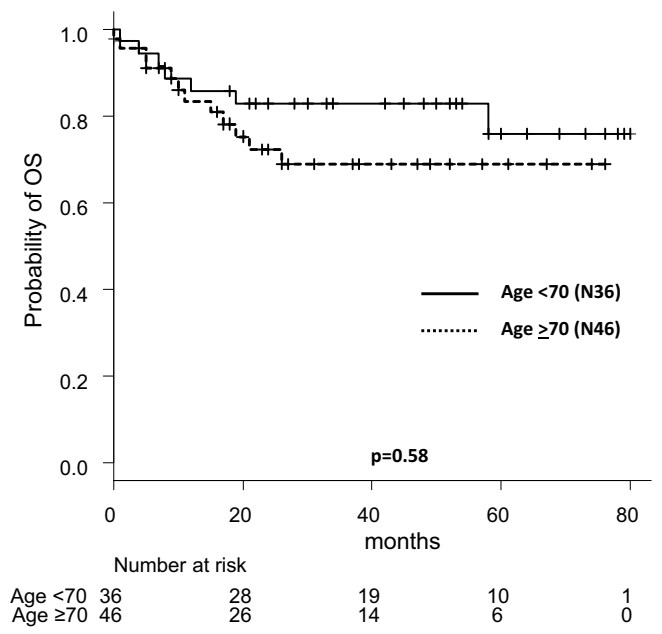

Figure 3. Overall survival according to patient age in each treatment group. (a) Overall survival according to patient age in the PHY group. (b) Overall survival according to patient age in the UNI group. PHY: physicians' decision group, UNI: unified dose-attenuation group

deaths (Supplementary material 3).

\section{Discussion}

Currently, DLBCL accounts for $>40 \%$ of all malignant lymphomas (ML) $(3,11)$, and it is the most frequent lymphoid malignancy in the United States and Japan. The ageadjusted incidence rates of DLLBCL have been reported to be 4.46 and 2.50 (per 100,000), respectively. In Europe, DLBCL ranked as the third most frequent lymphoid malignancy with an age-adjusted incidence of 3.13 (per 100,000) (1-3). Although a nationwide cancer surveillance program has not yet been established in Japan, a survey of $33 \%$ of the national population revealed annual increases in the incidence of ML by 3\% between 1993 and 2008, which might be predictive of further increases in the future (3).

Among elderly patients, DLBCL accounts for $>60 \%$ of all ML, and half of all patients with DLBCL are older than 65 years (1). Despite this frequency in the elderly population, however, chemotherapy for DLBCL is challenging in this population, as age-related changes are often observed in pharmacokinetics and pharmacodynamics, including absorption, diffusion, and metabolism or degradation of drugs, and they may be attributable to altered renal and liver functions $(7,16,17)$. As a result, increased drug toxicities may be responsive for insufficient treatment responses. Additionally, elderly individuals have impaired hematopoietic reserve capacities, which might result in more profound myelotoxicity and febrile neutropenia during chemotherapy (8).

In addition to toxicities, elderly patients also face an increased incidence of comorbidities. In a previous study of patients with aggressive NHL, 39\% of the patients aged $\leq 60$ years were found to have comorbid conditions, which apparently contrast with $69 \%$ of those aged $>60$ years; furthermore, the severity of comorbidities was a prognosticator of
OS, independent of the IPI (18). Additionally, the Charlson Comorbidity Index (CCI), which is used to evaluate the longitudinal risks of the patients with comorbid conditions (19), has been shown to correlate with age, such that patients aged 55-64 years had an average CCI score of 2.9, compared to 4.2 for those aged $\geq 75$ years (16).

In addition, age-related organ hypofunction, comorbidities, cognitive impairment, and poor nutrition status, may also be negative prognostic factors in this population. To this end, measures, such as the comprehensive geriatric index (CGI), which includes evaluations of comorbidity, cognition, nutritional status, psychological state, and social support, can be used to predict morbidity and mortality in elderly cancer patients (20).

In previous studies, the poor survival outcomes of elderly patients with DLBCL were attributed partly to chemotherapeutic dose reductions $(8,10)$, because the outcomes of fit elderly patients who were able to receive standard curativedose chemotherapy (3-week cycle R-CHOP) were found to be equivalent to those of younger patients $(9,10)$. When applicable, this standard regimen was shown to be highly effective even in the patients aged $>80$ years (21-23). By contrast, however, there was a tendency that attending physicians would withhold standard-dose chemotherapy for unfit or frail elderly patients, because the best supportive care was favored in such cases. This attitude might result in the extremely poor prognoses observed in this population (8). Although two trials of immunochemotherapy dose attenuation in unfit/frail DLBCL patients reported equivalent CR rates to those of fit patients, the OS rates were shorter in the former group $(13,14)$.

In a review article published in 2012, Fields et al. described an algorithm in which unfit patients were indicated to receive pre-phase therapy before proceeding to nonintensive chemotherapy or best supportive care depending on 
the likelihood of a transformation from frailty to fitness (4). Additionally, many previous reports described various methods used to select elderly patients might become fit to receive curative chemotherapy. However, if such decisions are made only based on an assessment of poor conditions at the time of diagnosis, patients with poor conditions caused by the ML itself might be excluded from receiving curative therapy. To address the above-stated concerns, we introduced the criteria of R-CHOP attenuation for DLBCL patients based on patients' age, PS, and damage to their renal and hepatic functions. These attenuation criteria were determined at our institute after a full review and discussion of standard pharmacology texts, pharmaceutical drug information, and data of published related studies and reviews retrieved from PubMed.

We analyzed the safety and efficacy of our attenuation system (UNI group), through comparisons with those of previous physicians' preference style (PHY group). Notably, we found that our system better preserved the administered sufficient doses and achieved high treatment efficacy without increases in toxicities. Accordingly, our system led to significant improvements in the CR and EFS rates, as well as a significant increase in OS among patients aged $>70$ years. Furthermore, patients in the UNI group had a longer treatment continuation interval, compared to those in the PHY group. We observed few violations of the selected attenuation criteria, as well as good applicability of the system. For example, 16 patients who could not initially receive RCHOP were administered high-dose dexamethasone prephase therapy, and 15 eventually proceeded to R-CHOP. In short, our dose-attenuation system, when combined with pre-phase therapy, was found to be applicable to the majority of patients with DLBCL, regardless of age, degree of organ damage, and/or PS.

In the PHY group, 6 patients received zero percent of cytotoxic agents. Five of them were in poor PS and poor general conditions. The attending physicians did not try to administer curative chemotherapy. For these patients, palliative care was chosen by their own eligibility criteria. However, if pre-phase therapies had been administered, and their general condition could be improved, then they might have proceeded to R-CHOP therapy. It is conceivable that if our unified attenuation system was applicable for these patients, they could have successfully received effective and safe chemotherapy.

It was considered meaningful to start treatment without curative intent and then to subsequently make decisions regarding the initiation of R-CHOP after achieving an improvement in organ damage, because the discontinuation or adjournment of chemotherapy might become needed in cases of newly developed renal or hepatic damage. As a result, our attenuation system could be applied safely and continuously to the majority of patients with DLBCL.

However, our study is also associated with some limitations. For example, the criteria for pre-phase therapy were unclear, and the effects of pre-phase therapy were not evalu- able in five patients who proceeded to R-CHOP after prephase therapy. Because, one patient died during R-CHOP, 3 patients were forced to discontinue R-CHOP because of toxicity, and one patient refused to continue R-CHOP. However, 10 other patients with severe organ damage and poor PSs could proceed to R-CHOP therapy after receiving clinical benefits from pre-phase therapy. Although new therapeutic agents considered to be less toxic than R-CHOP have been reported to be efficacious in basic and clinical trials, only a few have been made clinically available (24-26), and it remains unclear whether they are superior to doseattenuated R-CHOP.

Relative dose intensity (RDI) was not evaluated in each patient through all courses. The dosages of cytotoxic agents were calculated only in the first cycle. However, dosage of cytotoxic agents were adjusted by unified attenuation system in every cycles after induction of first cycle. Many patients could continue to receive the attenuated chemotherapies and attained both a good response and survival. This outcome might imply that a sufficient dose was administered safely throughout all courses.

As noted above, it may be beneficial to use objective measures such as the CCI or CGI, when selecting fit patients prior to initiating $\mathrm{R}-\mathrm{CHOP}$, rather than making similar decisions based on the physician's preference. However, it remains unclear whether it is better to select fit patients as candidates for curative therapy or to provide treatment without curative intent. Treatment with curative intent could place even fit patients at a higher risk of experiencing excessive toxicities. In our dose-attenuation system, the goal of therapy cannot be clarified at the start of pre-phase or reduced-dose therapy. The responses to these treatments required verification, through which subsequent strategies could be determined. Generally, decisions regarding chemotherapy for elderly patients with hematological malignancies may vary according to the patients' condition. The present study, which demonstrated the possible prognostic impacts of dose-attenuated R-CHOP therapy among elderly patients, suggests that this population may benefit through the assessment of the frailty or acceptability. The assessment of the frailty or acceptability includes, patient's age, degree of organ damage, and PS.

In this study, we retrospectively evaluated the outcomes of our recently implemented dose-attenuation program regarding R-CHOP therapy for DLBCL in a population of elderly patients and/or those with comorbidities. We found that our system was applicable to the majority of the patients, and its use could maintain an individualized and desirable dose, leading to improvements in the CR and EFS rates of all patients and in the OS rate in elderly patients. However, further prospective studies with large numbers of patients with DLBCL are needed to establish the optimal treatment regimen, dose-attenuation system, and riskstratification for elderly and/or comorbid patients.

The authors state that they have no Conflict of Interest (COI). 


\section{References}

1. Morton LM, Wang SS, Devesa SS, Hartge P, Weisenburger DD, Linet MS. Lymphoma incidence patterns by WHO subtype in the United States, 1992-2001. Blood 107: 265-276, 2006.

2. Sant M, Allemani C, Tereanu C, et al. Incidence of hematologic malignancies in Europe by morphologic subtype: results of the HAEMACARE project. Blood 116: 3724-3734, 2010.

3. Chihara $D$, Ito $H$, Matsuda $T$, et al. Differences in incidence and trends of haematological malignancies in Japan and the United States. Br J Haematol 164: 536-545, 2014.

4. Fields PA, Linch DC. Treatment of the elderly patient with diffuse large B cell lymphoma. Br J Haematol 157: 159-170, 2012.

5. Sarkozy C, Coiffier B. Diffuse large B-cell lymphoma in the elderly: a review of potential difficulties. Clin Cancer Res 19: 16601669, 2013.

6. Morrison VA, Hamlin P, Soubeyran P, et al. Approach to therapy of diffuse large B-cell lymphoma in the elderly: the International Society of Geriatric Oncology (SIOG) expert position commentary. Ann Oncol 26: 1058-1068, 2015.

7. Balducci L. Pharmacology of antineoplastic medications in older cancer patients. Oncology 23: 78-85, 2009.

8. Lyman GH, Dale DC, Friedberg J, Crawford J, Fisher RI. Incidence and predictors of low chemotherapy dose-intensity in aggressive non-Hodgkin's lymphoma: a nationwide study. J Clin Oncol 22: 4302-4311, 2004.

9. Coiffier B, Lepage E, Briere J, et al. CHOP chemotherapy plus rituximab compared with CHOP alone in elderly patients with diffuse large-B-cell lymphoma. N Engl J Med 346: 235-242, 2002.

10. Lee KW, Kim DY, Yun T, et al. Doxorubicin-based chemotherapy for diffuse large B-cell lymphoma in elderly patients: comparison of treatment outcomes between young and elderly patients and the significance of doxorubicin dosage. Cancer 98: 2651-2656, 2003.

11. Thieblemont C, Coiffier B. Lymphoma in older patients. J Clin Oncol 25: 1916-1923, 2007.

12. Tucci A, Ferrari S, Bottelli C, Borlenghi E, Drera M, Rossi G. A comprehensive geriatric assessment is more effective than clinical judgment to identify elderly diffuse large cell lymphoma patients who benefit from aggressive therapy. Cancer 115: 4547-4553, 2009.

13. Olivieri A, Gini G, Bocci C, et al. Tailored therapy in an unselected population of 91 elderly patients with DLBCL prospectively evaluated using a simplified CGA. Oncologist 17: 663-672, 2012.

14. Spina M, Balzarotti M, Uziel L, et al. Modulated chemotherapy according to modified comprehensive geriatric assessment in 100 consecutive elderly patients with diffuse large B-cell lymphoma. Oncologist 17: 838-846, 2012.
15. Kanda $Y$. Investigation of the freely available easy-to-use software 'EZR' for medical statistics. Bone Marrow Transplant 48: 452458, 2013.

16. Pal SK, Hurria A. Impact of age, sex, and comorbidity on cancer therapy and disease progression. J Clin Oncol 28: 4086-4093, 2010.

17. Wynne HA, Cope LH, Mutch E, Rawlins MD, Woodhouse KW, James OF. The effect of age upon liver volume and apparent liver blood flow in healthy man. Hepatology 9: 297-301, 1989.

18. Janssen-Heijnen ML, van Spronsen DJ, Lemmens VE, Houterman S, Verheij KD, Coebergh JW. A population-based study of severity of comorbidity among patients with non-Hodgkin's lymphoma: prognostic impact independent of International Prognostic Index. Br J Haematol 129: 597-606, 2005.

19. Charlson ME, Pompei P, Ales KL, MacKenzie CR. A new method of classifying prognostic comorbidity in longitudinal studies: development and validation. J Chronic Diss 40: 373-383, 1987.

20. Extermann M, Hurria A. Comprehensive geriatric assessment for older patients with cancer. J Clin Oncol 25: 1824-1831, 2007.

21. Thieblemont C, Grossoeuvre A, Houot R, et al. Non-Hodgkin's lymphoma in very elderly patients over 80 years. A descriptive analysis of clinical presentation and outcome. Ann Oncol 19: 774779, 2008.

22. Peyrade F, Jardin F, Thieblemont C, et al. Attenuated immunochemotherapy regimen (R-miniCHOP) in elderly patients older than 80 years with diffuse large B-cell lymphoma: a multicentre, single-arm, phase 2 trial. Lancet Oncol 12: 460-468, 2011.

23. Zinzani PL, Storti S, Zaccaria A, et al. Elderly aggressivehistology non-Hodgkin's lymphoma: first-line VNCOP-B regimen experience on 350 patients. Blood 94: 33-38, 1999.

24. Dasmahapatra G, Patel H, Dent P, Fisher RI, Friedberg J, Grant S. The Bruton tyrosine kinase (BTK) inhibitor PCI-32765 synergistically increases proteasome inhibitor activity in diffuse large-B cell lymphoma (DLBCL) and mantle cell lymphoma (MCL) cells sensitive or resistant to bortezomib. Br J Haematol 161: 43-56, 2013.

25. Park SI, Grover NS, Olajide O, et al. A phase II trial of bendamustine in combination with rituximab in older patients with previously untreated diffuse large B-cell lymphoma. Br J Haematol 175: 281-289, 2016.

26. Zinzani PL, Pellegrini C, Argnani L, Broccoli A. Prolonged disease-free survival in elderly relapsed diffuse large B-cell lymphoma patients treated with lenalidomide plus rituximab. Haematologica 101: e385-e386, 2016.

The Internal Medicine is an Open Access journal distributed under the Creative Commons Attribution-NonCommercial-NoDerivatives 4.0 International License. To view the details of this license, please visit (https://creativecommons.org/licenses/ by-nc-nd/4.0/).

(C) 2018 The Japanese Society of Internal Medicine Intern Med 57: 3521-3528, 2018 